\title{
The Recovery Experience: Stress, Recovery Capital, and Personal Views on Addiction and Recovery in Posttreatment Addiction Recovery
}

\author{
Therese C. Castillo \\ Seagulls Flight Foundation, Inc. \\ Ron Resurreccion \\ De La Salle University-Manila
}

\begin{abstract}
The study explored the experiences of individuals in posttreatment addiction recovery. More specifically, the research examined their experiences of stress, their recovery capital, as well as their personal views on addiction and recovery. Existing literature suggests that individuals with addiction require a substantial quality of recovery capital to overcome stress and challenges in all stages of recovery. The research adopted a case study design and interviewed eight participants on their experiences of posttreatment addiction recovery. Interview responses were analyzed through categorizing codes and themes and then submitting these for external audit. Results showed that the recovery experience in posttreatment addiction recovery included dealing with stress during reintegration to the mainstream environment, rebuilding relationships, and returning to daily functioning (getting a job, earning money, fulfilling responsibilities). Recovery connections, social support, and recovery-supportive activities were important factors in sustaining recovery. Addiction was considered as a lifestyle that provides temporary relief and hinders building meaningful relationships while recovery was depicted as an ongoing process requiring support and finding purpose in being in recovery.
\end{abstract}

Keywords: recovery, addiction, recovery capital, posttreatment, stress

Correspondence concerning this article can be addressed to Therese C. Castillo, Level 10-1, One Global Place, 5 th Ave. cor. 25th St. BGC Fort Bonifacio, Taguig City. Email: thereseccastillo@gmail.com. 
The media has portrayed people who use and sell drugs as both criminals and victims. How they are depicted in the news affects society's response, acceptance, and attitudes towards them (Bries, Marasigan \& Ngina, 2018). A person in addiction recovery is expected to encounter stress and challenges throughout the process and different stages of recovery. However, little is known about how stress is experienced in posttreatment (Laudet \& White, 2008). Recovering from addiction could be different in the Philippines because of the impact of the government's recent approach to eradicating drug use. A recent study showed that recovering individuals may face challenges of discrimination in finding work and gaining access to services because of the increasing stigma that surrounds drug use and addiction (Labor, 2018). This study explored what it is like to be in recovery, the challenges in the process of change, and the resources needed to sustain recovery.

\section{The Recovery Process}

Reintegration to mainstream society is part of the recovery process for people with substance use disorders. This is what is being referred to as "normal" when an individual in recovery moves towards aftercare or posttreatment (Duffy \& Baldwin, 2013). Normal means to gradually go back to daily functioning and lifestyle such as staying in a decent home, working, earning money, making friends, and spending time with family. For recovering persons to achieve these goals and walk through these steps, they need substantial recovery capital to handle the pressures and stress experienced in posttreatment. Recovery capital is an individual's source of strength and motivation in recovery. It includes social networks, education, employment, financial assets, health, beliefs, and values. Recovery capital may increase over time as the person progresses in recovery (Duffy \& Baldwin, 2013).

\section{Stress and Recovery}

Stress is a part of recovery, as it is part of daily life. However, stress may be particularly experienced in posttreatment in the form of pressures to do "more" and to make up for "lost time" during active 
addiction. Stress is one of the major factors in a person's ability to sustain recovery. A person who may be unable to handle stress after being in treatment can move towards a relapse to substance use rather than to staying in recovery. Given that addiction is a chronic brain disease, there are challenges with managing emotions and impulses in the limbic system (National Institute of Drug Abuse, 2018). This pressure can become too overwhelming as an individual takes on too many activities too soon. In this study, posttreatment addiction recovery stress was defined as the pressures experienced by the recovering person after completion of formal treatment.

\section{Recovery Capital}

Both personal resources and social capital are significant to sustaining recovery and overcoming obstacles that are encountered on a day-to-day basis. Social support plays an important role in sustaining recovery (Mawson, Best, Dingle, \& Lubman, 2015). Building relationships with other individuals who are in recovery for support and care is an important factor as well (Johansen, Brendryen, Darnell, \& Wennesland, 2013). It may be possible that one of the reasons social recovery capital takes a more meaningful role in this stage is because of the process of giving and receiving help as recovering people. People in recovery feel more connected with other people in recovery and tend to have the desire to spend most of their time with them instead of those not in recovery (Melick, McCartney, \& Best, 2013). Connectedness and universality are achieved when a person in recovery engages in support group meetings and activities.

Change can be overwhelming to an individual in recovery, even after completing treatment and acquiring necessary knowledge and skills to cope. During active addiction, substances may have been used to manage emotions and to cope with uncomfortable feelings. However, a person in posttreatment addiction recovery should have gained enough foundational skills to regulate emotions and practice resilience.

Recovery involves a process of healing and discovery beyond addressing symptoms of addiction (White, Boyle, \& Loveland, 2004) and every individual's experience in recovery is very personal and 
unique. Given this, it is important to consider not just the person's experiences but their personal views on addiction and recovery. This study presumed that a person in recovery is in the process of change, ideally in the maintenance stage in posttreatment addiction recovery. Being in the maintenance stage of change means that the person in recovery has made a decision to sustain recovery and is committed to maintaining this change (Nidecker, DiClemente, Bennett, \& Bellack, 2008). However, being in the maintenance stage does not take away the experience of stress in the process. Lapse or a relapse to substance use is always a possibility even as the person tries to protect their recovery. As an individual moves through the process, their understanding of their addiction and recovery creates an impact on their attitudes and behaviors. In this study, the reality of recovery being a continuous process of ups and downs is emphasized.

Addiction is a chronic disease of the brain, which is demonstrated by intense cravings leading to drug seeking, destructive, and catastrophic consequences. It is comparable to other chronic diseases because of the changes in the brain that affect behaviors of persons who abuse drugs (National Institute of Drug Abuse, 2018). The perspectives on addiction and how it is interpreted presents diverse views influenced by society, culture, and science over time (Hammer, Dingel, Ostergren, Nowakowski, \& Koenig, 2012). Throughout the years, recovery has evolved as a construct that guided efforts to professionalize addiction treatment.

The present study sought to contribute to the understanding of recovery experiences after treatment in the Philippines. It is focused on the posttreatment completion period where there is reduced supervision from treatment providers and which required the individual's conscious efforts to protect and sustain recovery. This is essentially within the identified critical time before reaching a recovery benchmark of five years. The current research aimed to explore and understand the experiences of stress among people in posttreatment addiction recovery, their recovery capital, and their personal views of addiction and recovery. 


\section{METHOD}

\section{Sampling}

This research utilized purposive sampling to best understand and explore the experiences of eight participants (one female and seven male) in posttreatment addiction recovery. The youngest was 31 years old and the oldest was 58 years old. The youngest participant was admitted to treatment at 17 years old and the oldest was admitted to treatment at 49 years old. The average age was 28 years old. All participants received formal education, up to at least a bachelor's degree, except for one participant who finished high school. Six out of eight participants were self-employed working in different areas of business. Five out of eight participants were addicted to methamphetamine while others were addicted to other types of stimulants and depressants. All participants except for one have been in recovery for at least 10 years. All participants underwent residential treatment for addiction to substances.

\section{Instrument}

The interview guide contained a set of open-ended interview questions focusing on the following variables: personal views on addiction and recovery ("Since you have finished formal treatment, can you tell me about your experience with recovery?”), stress experienced in posttreatment addiction recovery ("What are situations that seem to be overwhelming at this stage in recovery?”), and recovery capital in posttreatment addiction recovery ("How are your personal strengths and skills helpful at this stage in your recovery?”). Interview questions focused on the respondents' current state of recovery. It was validated by three experts in research that possess a doctorate degree in psychology. Two of the validators were involved in the addiction treatment field. All validators were provided with a research summary and a validation form containing the complete set of interview questions. The validation form was to be provided with comments to accept, reject, or revise each question. The consolidated input from the three validators was the basis for finalizing the interview guide. 


\section{Procedure}

Before data gathering, approval was acquired from the University Ethics Committee. The researchers identified the participants according to the criteria assigned and conducted one-on-one interviews using an in-depth interview guide on the experiences of people in posttreatment addiction recovery. Individual interviews were scheduled according to the most convenient time available for the participants. They were held in a private session room that was conducive for a comfortable conversation.

Each participant reviewed and signed the participant consent form that clearly stated the purpose of the study, expected duration of interview, how results will be used and published, and terms and guidelines on confidentiality. The consent form also explicitly stated that at any time the participant feels distressed with the interview process, he or she has the right to withdraw from the research. The participant was also provided with contact information on available support for debriefing and intervention.

Interviews were audio/video recorded. The researchers noted observations for additional data. Probing was done during the interview as necessary and according to the willingness of the participant to disclose information. All responses and information exchanges were kept private, confidential, and for research purposes only. At the end of the interviews, the accuracy of the interview responses underwent member checking by asking the participants if the summary of responses had been accurately recorded and interpreted. The interview responses were transcribed per participant at the end of each interview.

\section{Data Analysis}

This study used qualitative thematic analysis (Braun \& Clarke, 2006) to analyze the data. Interview responses were transcribed and marked individually per participant. Each participant was assigned a code name to protect and maintain confidentiality. The transcripts, demographic sheets, and notes were collected and organized.

In conducting data analysis, interview responses were first assigned codes for every participant. Significant codes were then taken 
from the first level of content analysis on stress, recovery capital, and personal views on addiction and recovery. Themes were identified for every participant according to research question. A cross analysis was conducted to identify the emerging themes. The researchers served as the coders while an external auditor, who has experience in qualitative research and holds a master's degree in psychology, reviewed the themes to ensure consistent results, reduce possible bias of researchers, and maintain objectivity. Themes were finalized through consensus among the coders and the external auditor.

\section{RESULTS}

The results include emerging themes on 1) Experiences of Stress in Posttreatment Addiction Recovery, 2) Recovery Capital, and 3) Personal Views on Addiction and Recovery (see Figure 1). The succeeding section describes each themes and presents the narratives of the participants.

\section{Stress in Posttreatment Addiction Recovery}

Reintegration to the mainstream environment. Participants shared that they experienced stress in the process of reintegration or transition from addiction treatment to normal activities and settings. There was a period of adjustment to going back into roles and responsibilities at home, work, and school. The challenges included dealing with exposure to triggers in school, unemployment, and making up for lost time. For all participants, this transition was a major source of stress.

Dealing with people and social situations. This involved meeting with old friends from the time of active addiction, dealing with and talking about their views on addiction treatment, personal disclosure about coming from treatment, and saying no to friends and managing peer pressure. Several times, they had to answer the question, "Where have you been?" One of the respondents stated, "For me it's that. Also in line with trust, being able to say no to parties, anything that would put me in danger." 


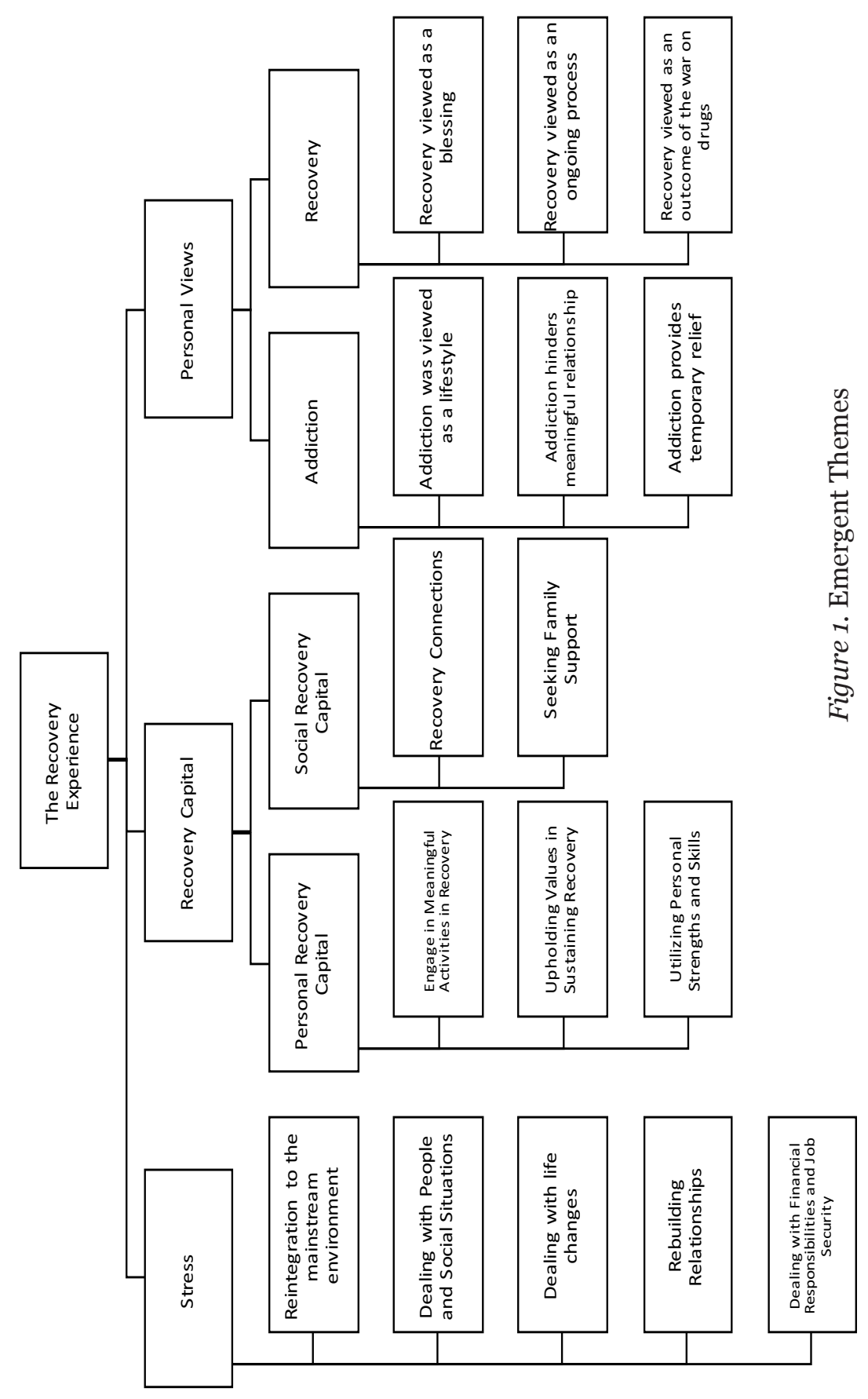


Dealing with life changes. For the respondents, adjusting to developmental changes while recovering also caused stress. Some respondents had to adjust to married life and parenthood, both of which entailed a lot of responsibilities. One respondent even had to deal with unplanned pregnancy. One respondent shared his experience, “... when I got married. Actually, before we got married, she got pregnant first. So at that time, I just graduated and I still don't have work." Others experienced death in the family. Another respondent dealt with assuming the role of a provider for the family when the mother passed away and shared, "I was an only child. That was the most stressful for me. I had to think about where to get money to provide."

Rebuilding relationships. Given that addiction had a negative impact on relationships, being in posttreatment addiction recovery meant rebuilding relationships. There were challenges and stress involved in reestablishing the trust and connections within and among family members. Unresolved issues of family members were an impediment to trying to build healthy relationships. One recoveree said,

I was afraid of my daughter because my daughter didn't welcome me yet. She didn't want anyone to know but she's seeing a shrinkand the shrink told her enough is enough. My daughter wanted to continue living with my parents instead of coming home with me. That was the stress.

Dealing with financial responsibilities and job security. Ensuring adequate financial resources was one of the most stressful experiences coming from treatment. The recoverees had to deal with financial crisis, pressure of providing for the family, managing a start-up company, as well as harboring feelings of insecurity due to unemployment and role reversal with partner. One of them shared, "I found out that she was actually borrowing money to buy milk for my kid. That's why it was more stressful for me.” Another recoveree added, "The business hit a crisis and I felt very isolated and then it came to a point that even my wife didn't believe anymore that I could recover from that really bad situation." 


\section{Recovery Capital in Posttreatment Addiction Recovery}

Personal recovery capital. This refers to efforts of recovering individuals to stay away from addiction and sustain recovery. This included engaging in meaningful activities, strengthening personal values, and using strengths and skills.

Engaging in meaningfulactivities in recovery. Recoverees joined activities that provided a sense of meaning and purpose for their recovery. These activities were focused on being employed in fulfilling work, spending quality time with family, engaging in leisure activities, and enriching their relationship with a higher being. These activities were considered to be the most important to individuals in recovery. These gave a sense of purpose in staying in recovery. One of them stated, "When I'm playing golf...everything's just really for the moment. I see myself improve when I'm in golf. There are bad days, good days, and I have to be a good sport."

Upholding personal values in sustaining recovery. During the recovery period, recovering individuals leaned on their personal values to resist temptations. These values included being contented with a simpler lifestyle, keeping the faith in a higher being, strengthening relationships and regaining trust, helping others, loving and caring for the self, and commitment to recovery. These values were a major contributor in staying in recovery. As shared by the participants, "I saw my family-how I gained back their trust to me. Those are motivations that make me drug-free" and "Of course you don't want to throw all those away. You've built your relationship already, your kids, your family."

Utilizing personal strengths and skills. In the process of recovery, the individual acquired and developed personal strengths and skills such as determination, good judgement, optimism, humility, resilience, and ability to resist temptation. These individual factors were instrumental in dealing with daily activities, overcoming challenges, and protecting recovery. One respondent expressed, "I encounter stress almost everyday but instead of giving up, I made sure that I always think positive. Because that's one thing I learned inside the facility. I don't blame the one who is giving me stress." Another respondent described his triumph in overcoming his weakness by 
saying,

Because unlike my other colleagues when they go back to the outside-to reality, they encounter stress. To manage the stress, they go back to drugs. I would never want to-I hated drugs already. My strength was I really made it a point to succeed.

Social recovery capital. This refers to resources outside the self such as friends, fellow recoverees, and family members.

Recovery connections. This included receiving support from the treatment facility, volunteering in recovery activities, joining support groups, maintaining relationships with recovery peers and the recovery community, having senior peersin recovery, rolemodeling with other recovering people, and giving back to the recovery community. According to one participant, "I guess it would be to encourage people. I feel that it is something that I can give. I guess my biggest asset is I can give hope to others" and "I've always used my relationships in church and in recovery so, it's really about having support." However, connecting with peers also led to challenges. Staying connected with the right friends who can positively influence the person in recovery required effort. More so, having to stay connected with friends who were still actively using drugs was difficult. "I have friends who, up to this day, go to casinos and there are instances when some of your friends invite you. But since you know you're a special case, you try to avoid."

Seeking family support. The family was a strong contributor to sustaining recovery. Most of the respondents believed that their experiences with their families provided them strength during difficult times. Their families became a great source of motivation, guidance, unconditional love, and joy. One respondent claimed, "My family and kids. I don't think I would be able to survive it without those and of course good communication." Some respondents also expressed that their families were instrumental in providing them with basic needs during the early part of their reintegration. Three respondents experienced being financially supported after treatment and did not experience any problems with physical resources. One of them expressed, "Well, I can say I'm also very lucky because without our parents, we can't do this alone." 


\section{Personal Views on Addiction in Posttreatment Addiction Recovery}

Addiction was viewed as a lifestyle. For most respondents, drug use was already part of their daily routine and so stopping it caused anxiety. According to one recoveree, "Addiction means you feel uneasy and you don't feel confident if you are not using the substance... I could not survive without shabu even for a minute that time."

Addiction hinders meaningful relationship. Because addiction affects emotional and cognitive functioning, it can impair a person's ability to establish good relationships. Most of the participants' interactions with other people did not lead to genuine relationships and were purely transactional. One of them stated, "I never thought of having long-term or substantive types of relationships. They were more of what I could get out of those types of relationships in the least amount of time."

Addiction provides temporary relief. Recoverees described the positive and pleasurable feelings from taking the abused substances. Some respondents claimed that they used these substances to keep them awake and alert so that they will be more productive at work. Using substances with fellow individuals who use drugs also gave them a sense of belongingness. However, prolonged use of substances led to loss of control. One of them described, "I was powerless already. It came so fast...Something that's uncontrollable. Most of my decisions were mostly made to satisfy that addiction."

\section{Personal Views on Recovery in Posttreatment Addiction Recovery}

Recovery viewed as a blessing. For most respondents, being able to recover from addiction was a blessing. It gave them an opportunity to have a healthier lifestyle and live a life free from substances. Their recovery also allowed them to develop positive qualities and reestablish relationships. Recovery brought them happiness and a better life perspective. One respondent expressed, "Happy, living with my family, and everything. Yeah, so it's actually everything." 
Recovery viewed as an ongoing process. Respondents considered themselves to be "recovering" when they had an increased awareness of their addiction and were equipped with tools and strategies to manage recovery. Recovery is taken a day at a time where there are no guarantees in maintaining progress. Support was emphasized to be an important aspect in sustaining recovery. Recovery maintenance activities were also acknowledged to be significant to protecting recovery. Because of addiction's nature and recovery's continuous process, getting involved in activities such as attending support groups was essential. One of the recoverees said, "It's ongoing. That is why it is important that you do not disconnect from the support group that helped you recover."

There are different aspects to sustaining recovery and abstaining from substances was only one of it. The other facets included mental health, physical health, behaviors, productivity, individual factors, and more. One of the respondents stated,

It's essentially a health issue. Stopping use is just maybe a small portion of the recovery process. It's also recovery in its fullest sense. Meaning, you have to be restored to (the) original design of who you are as a human being, essentially.

Finding purpose in being and staying in recovery was also important. Being in recovery meant realizing the importance of family, appreciating the self, changing one's outlook in life, experiencing joy and inner peace, understanding existence and the sense of being in recovery, making treatment work, and improving the quality of life. One of them shared, "There's like a fulfillment that I'm staying on that path and also the awareness that most of the struggles that I have are of normal people."

Recovery viewed as an outcome of the war on drugs. Based on the respondents' experiences, they expressed that the government's war on drugs paved the way for the recovery of many people who use drugs. Given the enormous problem of drug use in the country, most of them saw the current campaign as a necessary step to solve it. However, there were also those who explained that although the intent is positive, there are differences in recovery potential of those in middle to high socio-economic classes and those with a low 
socio-economic classes. Another said that only those in the middle to upper classes are privileged to have access to quality treatment. One of the recoverees explained,

Those of us who are in the middle or upper class, treatment is easier to avail. I guess it's economic also. Because those of the poorest of the poor, they don't really have that equal opportunity to get good treatment.

One recovering individual expressed his disappointment with society's negative response to how the government is addressing the drug problem. To him, the methamphetamine problem does not require the measures the police are taking. Some respondents narrated their ability to contribute to the campaign through forming Narcotics Anonymous meetings, advocacy groups, being role models in recovery, and showing that being in recovery is good and that those who recovered can be as productive as anyone. Quality treatment was viewed as a requirement to initiating recovery for the people affected by the war on drugs campaign. One participant expressed,

It's good to be a living example that there's a solution that can be done. I think it's good to show others, especially the drug dependents that it's possible-that you can be at par with anybody in any place, at work, in school.

\section{DISCUSSION}

For recovering individuals, transitioning from treatment activities to doing normal activities of daily functioning was a process that induced stress and pressure. This is a phase of adjustment for a person in recovery. According to Duffy and Baldwin (2013), for an individual in posttreatment addiction recovery, reintegration to the mainstream is movement towards normal daily functions.

The results are in line with literature in terms of experiences of change at this stage in recovery. Substantial recovery capital is required to manage stress in this process of transition (White, 2004). The respondents in this study were privileged to have a generally high recovery capital to help manage stressful events. They shared experiences of dealing with changes in their environment, dealing with 
people, finances, and productivity. These are in line with the study of White (2004) that identified daily functioning activities like dealing with friends, earning money, and spending time with family as part of going back to normal activities. Although the activities seem to be neutral and even positive, the experience of initiating and engaging in these activities is considered to be stressful. What was different was how people in recovery dealt with stress and pressures compared to when they were actively using substances.

Theresults suggest that overcoming stress, dealing with unexpected life events and changes, facing challenges in rebuilding relationships, and dealing with financial and job crises are instrumental in building strength in recovery. Stressful experiences do not just involve using substances in posttreatment recovery but also dealing with the different aspects of life to get back to regular functioning. The stressful events at this stage of recovery are perceived as challenges and opportunities for growth. However, developing this mindset requires time, experience, and effort, and involves struggles in early recovery.

The first five years of recovery after treatment are identified to be the most stressful time as it is a transition from intensive support from treatment and focusing on treatment activities alone to building a life in recovery with less support from treatment and increased personal accountability. As supported by literature, more recovery capital is needed to enable individuals to handle stress during recovery (Laudet \& White, 2008).

As mentioned in the earlier sections of the research, recovery capital increases over time in treatment and through the process of recovery (Mawson et al., 2015). The respondents in the study had been in recovery for at least seven years, which meant they had a longer period to acquire and develop resources for recovery. One of the distinct factors that seemed to have contributed to sustaining a good recovery capital was socioeconomic status. The results may have been different if the participants came from a low socioeconomic status and had more limitations and barriers in accessing money, jobs, and stable homes.

Just as literature has presented, social connections and engaging in recovery-supportive activities are significant factors in sustaining recovery. People in recovery are able to gain insight on their personal 
journey from interactions with other people that are in recovery as well. Having mentors and peers in recovery has been helpful in managing stress and challenges. It may be understood that similar experiences can bring people together to build hope from and for each other. The concept of role modeling and helping other people was found to be a source of motivation in choosing to stay in recovery. As presented by a similar study, being surrounded by peers, getting involved in meaningful activities and gaining a longer time in recovery allows improved daily functioning (Best et al., 2012; Melick et al., 2013).

Getting involved in activities that are meaningful and rewarding, spending time with family, prayer, and playing sports are activities that people in active addiction lose as they progress deeper in substance use. During active addiction, a person immersed in drug use becomes isolated from the world and eventually loses sight of what is important, special, and meaningful. In the process of recovery, the person gradually moves towards meaningful connections through people and activities. Being in posttreatment addiction recovery, there is greater value for quality relationships and taking care of the self. At this stage, people seek activities that are meaningful and valuable to their recovery.

Moreover, values have been found to be evident in sustaining recovery. It seemed that values are developed as a person goes through the stages of change, and gains insight on and awareness of his addiction and recovery. Values are renewed, relearned and discovered in the process. They significantly contribute to making decisions and choices related to staying in recovery and moving towards activities that promote protecting recovery. Personal recovery capital including personal strengths and skills to manage recovery and overcome stress are foundational skills needed in regulating emotions and developing resiliency (White et al., 2004).

Results reveal difficulties in developing new friendships as well as maintaining friendships with those who are still actively using substances. Friendships are mostly focused within the recovery circle after treatment although participants gain new peers at work and school settings after some time. This is consistent with literature that suggests most friendships are primarily within the recovery community and become a strong source of support (Duffy \& Baldwin, 
2013). Friendships within the recovery community offer safety, universality, and practical assistance with issues exclusive to being "recovering people."

Results likewise reveal that family support is helpful in sustaining recovery, consistent with literature that family is an external source of motivation (Duffy \& Baldwin, 2013). Having a healthy relationship with the family becomes a strong source of support. However, if the relationship is unhealthy and destructive, exposure to family members can also be a source of stress. It is important to note that family relationships need time, mutual efforts, and restructuring of dynamics as the person moves from active addiction to recovery.

One form of family support is providing physical resources for persons in recovery to restart lives without worrying about money, housing, and transportation. In this study, family members willingly provided or assisted with living arrangements after treatment. This is different from what goes on in developed countries where such support is usually provided by the government (Mericle, Miles, \& Way, 2015). A possible explanation for this divergence in findings is cultural differences as Filipinos have a tendency to take care of their children even as adults, whether it be financial or emotional support.

Strength in recovery was instrumental to overcoming challenges and stress and managing daily life situations. Unlike being in active addiction, persons in posttreatment addiction recovery become equipped with skills and acquired strength in making decisions directed to sustaining recovery. The experience of addiction was described to be progressive over time and marked by losing interest in activities that the person used to be involved in. The lifestyle was focused on seeking and using substances despite the negative consequences of addiction (e.g., in the areas of daily functioning, social relationships, mood and behavior, as well as cognitive functions). This is in accordance with literature on addiction and how the brain loses its ability to manage impulses and emotions in the limbic system (National Institute of Drug Abuse, 2018). The experience was described as a loss of power and control with everything being motivated by substance use. Having an awareness that addiction is a chronic brain disease provides an understanding of why behaviors and attitudes were directed towards drug-seeking and use despite the harm it can cause to the self and 
others.

When persons with addiction engage in and sustain recovery, this is the time when things become clearer to them, including what they have lost along the way. At the onset, initial effects of substance use are remembered to be positive. However, as time passes, people in posttreatment addiction recovery have stronger memories on the negative impact addiction has caused in their lives. There appears to be fear in going back to the life of addiction. Addiction is depicted to be a time of isolation while being in recovery is about gaining connection.

The results further suggest that the experience of being in recovery in posttreatment is a continuing and life-long process. There are no guarantees that people will avoid lapses or not return to addictive behaviors. Recovery in general is a continuous and an ongoing process (Sheedy \& Whitter, 2009). The only difference with those coming from formal treatment is that there is an increased awareness of the self, addiction, and recovery. Compared to being in formal treatment, the support from the treatment provider is more intensive in posttreatment. After treatment, adapting to changes and sustaining recovery becomes challenging. At this stage in recovery, engaging in recovery-supportive activities is a way of maintaining progress. Support may be received from family and/or the recovery community. Great value is placed on connections where it be with family or the recovery peers.

It was recognized that recovery brings positive consequences and improvements in the quality of life. However, the great rewards of recovery are not immediate and expectedly gained after dealing with quite an amount of stress over time. Unlike how a substance provides immediate positive effects, rewards of recovery take a longer time to realize and take advantage of. If a person in recovery is unable to manage stress in a healthy manner with required support, a relapse to substance use is more likely to happen.

Based on the respondents' experiences, Operation Tokhang is positive given the enormous problem of drug use in the country. Despite this, there are differences in recovery potential for those from a middle to high socioeconomic status and those from a low socioeconomic status. For the participants, only those in the middle to upper classes have access to quality treatment and this might be a factor that protected them from the aggression being shown towards 
people who use drugs. In this day and age in the Philippines, recovering is critical to one's safety especially for those from a low socioeconomic status. However, the lack of physical recovery capital like finances and housing are critical sources of stress, which will pose difficulties in improving other areas in life and sustaining recovery.

Despite what is known about addiction and recovery, societal attitudes reflect stigma and discrimination towards people who are involved with drugs. A recent study in the Philippines described how society associates feelings of anger and hate with people who use drugs. It also reported that increased emotions of anger and hate meant denial of human nature and human uniqueness for people who use drugs (Labor, 2018). As discussed earlier, media has portrayed sellers and users as criminals and victims. The more frequent the negative portrayal of addiction, the more negative the attitude of society towards them (Bries et al., 2018). As the media shows more and more images of people who use drugs as "criminals," the more hatred is shown towards them.

However, news that show people who have used drugs as victims and have eventually changed their lives lead to a positive response and attitude from viewers. This further emphasizes that the way the media portrays people with addiction and recovery affects how society understands and accepts them (Bries et al., 2018). This stigma is likely why most of the participants still chose not to be open about being in recovery for fear over their safety, possible negative impact on business, and negative reactions from colleagues. The war on drugs campaign may be encouraging efforts to address drug use but understanding recovery means acceptance of lapses to substance use as a reality. The campaign can promote unrealistic expectations from people who engage in treatment and are in recovery.

Some respondents narrated their ability to contribute to the campaign through support groups, advocacy, being role models, and showing that being in recovery is good. Based on their experience, treatment was viewed as necessary to initiate recovery for the people affected by the war on drugs campaign.

There are several facets of recovery and not taking drugs and substances is not enough. Finding the purpose in being and staying in recovery is also significant in the process. After a series of negative 
experiences during addiction, the person in recovery after finishing treatment strives for bigger goals. The efforts to sustain recovery do not end when treatment ends. Thus, the results of the study may be valuable to developing chronic-care treatment models rather than following an acute-care model. Understanding the recovery experience after a person completes treatment allows better insight on what may be provided for continuity of the recovery progress and recovery capital the person has gained over time. An important consideration is that the respondents in this study came from an intensive residential treatment with two levels of care (primary care and aftercare). The information may be valuable to case management programs and treatment professionals working in the addiction field. Coordination among recovery-support services are usually fragmented and gaining better understanding of how a person in recovery walks through stress, what type of resources are helpful and how he views his addiction and recovery may be appreciated. The hope and success the respondents have experienced in the process may be valuable to Filipinos in terms of understanding that recovery is possible and addiction is treatable.

The information presented in the study may provide greater awareness of the capabilities and strengths of people in recovery but this is only possible with quality support from family, friends, and community. Recovery is a process and the experiences of stress in posttreatment are critical components in developing personal recovery capital and other forms of recovery capital. Substantial recovery capital is required to manage stress experiences and develop a deeper understanding of what it is like to have an addiction and what it means to be in recovery.

The study provides an understanding of recovery at a specific stage of recovery: posttreatment, a critical time in sustaining recovery with decreased support from the treatment provider and increased responsibilities. Posttreatment is also a stage when expectations from family members and loved ones are possibly high and there is pressure to maintain progress from treatment. This study provides evidence that recovery after treatment involves experiencing crises, dealing with unpleasant experiences with people, possibilities of lapse to substance use, and having to deal with these experiences without the use of substances. It also highlights the importance of family in the Philippine 
setting. Family is a positive source of support when relationships and behaviors encourage recovery. It provides perspective on recovering during the war on drugs campaign when social stigma against people with addiction is high and expectations to maintain recovery may be unrealistic.

Formal treatment is only the foundation of initiating and engaging in addiction recovery. Sustaining recovery in posttreatment poses threats and pressures that may lead to lapse to substance use when there is not enough support. Situations such as financial crisis, relationship conflicts, and unemployment are critical areas for a recovering person that require external social support apart from individual strengths and skills. Recovery is continuous and ongoing. There is a need to consciously make efforts to build recovery capital, capitalize on strengths, and manage the deficits in recovery. Gaining awareness of the purpose of staying in recovery is one of the most valuable aspects of choosing to be in recovery. It may be for one's family or simply for a new appreciation of life. An individual in recovery becomes more resilient amidst stressful experiences with the help and support of the recovery community and substantial recovery capital.

\section{Limitations of the Study}

The method of gathering data in this research was dependent on the quality of interview responses. The data was also dependent on the honesty, candidness, and level of self-disclosure of the participants on their experiences in recovery. The sensitivity of the research topic may have affected the participants' willingness to be truthful and candid. To mitigate this, participant guidelines were discussed prior to the interview to level expectations and promote emotional safety. The method of interviewing was non-threatening, informal, and set in a private room to encourage participants to disclose comfortably. The researchers practiced techniques such as establishing rapport, reflective listening and unconditional positive regard.

Moreover, validation of sustained recovery status of participants was limited to the recommendation of the treatment provider. Further validation from family members or close relatives and friends was not conducted. Although the interviews were personal and intimate 
in nature, the researchers maintained objectivity by following the validated interview guide, conducting member checking with the participant, and subjecting the results for external audit.

Gender is another limitation since there was only one female among eight participants. There may be differences in the recovery experience if genders were balanced in number. Likewise, most of the participants in the sample received a high level of formal education (minimum of high school) and came from a middle to upper middle socioeconomic status. Their access to physical recovery capital is likely different from those from a lower socioeconomic status.

\section{Implications for Research and Practice}

The researchers recommend a more diverse sample to provide different perspectives coming from various types of treatment. Focusing on a specific level of care, post-primary care program or postaftercare program, can also be done to provide a focus on the gaps and strengths in transition from one level of care to another. Level of care, recovery capital, and quality of life may also be a potential quantitative research topic. Looking into what makes a supportive family in recovery is also suggested. Since one of the respondents had negative experiences with the way their family provided support, it may be valuable to explore what are recovery-supportive behaviors of a Filipino family. A quantitative study on the association between socioeconomic status and recovery potential in the context of the war on drugs campaign is also recommended for further studies.

Based on the results of the study, the researchers recommend to practitioners handling clients with substance use disorders to understand recovery as a process and provide enough guidance for transition from treatment to the mainstream. It is also suggested to work with recovery-support systems to assist in the transition as the client moves back into various roles. According to the results, a client may benefit most from collaborative efforts from the treatment provider, family, and support group systems. As a practitioner, it may be best to encourage teamwork and therapeutic alliance among possible sources of support through efficient case management. This may be applied to treatment planning, drafting discharge reports, and 
continuing care plans to facilitate transitions smoothly and effectively. Applying a chronic-care treatment model rather than promoting acute-care approaches is recommended to be practiced by different treatment providers and support systems.

\section{REFERENCES}

Braun, V., \& Clarke, V. (2006). Using thematic analysis in psychology. Qualitative Research in Psychology, 3(2), 77-101.

Bries, F., Marasigan, J., \& Ngina, K. (2018). The news shapes our views: Media exposure and attitudes towards drug addicts. Paper presented at the meeting of of the PAP Taskforce on Drug Recovery Support, QCX, Quezon City.

Hammer, R. R., Dingel, M. J., Ostergren, J. E., Nowakowski, K. E., \& Koenig, B. A. (2012). The experience of addiction as told by the addicted: Incorporating biological understandings into self-story. Cult Med Psychiatry, 36, 712-734.

Johansen, A. B., Brendryen, H., Darnell, F. J., \& Wennesland, D. K. (2013). Practical support aids addiction recovery: The positive identity model of change. BMC Psychiatry, 13(1), 201.

Labor, P., (2018). Why drug sellers and users are not seen as human. Paper presented at the meeting of of the PAP Taskforce on Drug Recovery Support, QCX, Quezon City.

Laudet, A. B., \& White, W. L. (2008). Recovery capital as prospective predictor of sustained recovery, life satisfaction and stress among former poly-substance users. Subt Use Misuse, 43(1), 27-54.

Mawson, E., Best, D., Dingle, G. A., \& Lubman, D. I. (2015). Social identity, social networks and recovery capital in emerging adulthood: A pilot study. Substance Abuse Treatment, Prevention, and Policy, 10(45), 1-11. doi:10.1186/s13011-015-0041-2.

Melick, M., McCartney, D., \& Best, D. (2013). Ongoing recovery support and peer networks: A preliminary investigation of recovery champions and their peers. Journal of Groups in Addiction \& Recovery, 8(3), 185-199.

Mericle, A., Miles, J., \& Way, F., (2015). Recovery residences and providing safe and supportive housing for individuals overcoming 
addiction. Journal of Drug Issues, 45(4), 368-384.

National Institude of Drug Abuse. (2018, July 20). Drugs, brains, and behavior: The science of addiction. Retrieved from https://www. drugabuse.gov/publications/drugs-brains-behavior-scienceaddiction.

Sheedy, C., \& Whitter, M. (2009). Guiding principles and elements of recovery-oriented systems of care: What do we know from the research? Nova Science Publishers, 9(4), 225-286.

White, W. (2004) Transformational change and addiction recovery. Counselor, 5(4), 30-32.

White, W., Boyle, M., \& Loveland, D. (2004). Recovery from addiction and recovery from mental illness: Shared and contrasting lessons. In R. Ralph \& P. Corrigan (Eds.), Recovery and mental illness: Consumer visions and research paradigms (pp. 233-258). Washington DC: American Psychological Association.. 